\title{
Premature chromosome condensation reveals DNA-PK independent pathways of chromosome break repair
}

\author{
GEORGIA I. TERZOUDI ${ }^{1}$, SATYENDRA KUMAR SINGH ${ }^{2}$, GABRIEL E. PANTELIAS ${ }^{1}$ and GEORGE ILIAKIS ${ }^{2}$ \\ ${ }^{1}$ Institute of Radioisotopes and Radiodiagnostic Products, National Centre for Scientific Research 'Demokritos', \\ 15310 Ag. Paraskevi Attikis, Athens, Greece; ${ }^{2}$ Institute of Medical Radiation Biology, \\ University of Duisburg-Essen Medical School, D-45122 Essen, Germany
}

Received March 21, 2008; Accepted May 9, 2008

DOI: 10.3892/ijo_00000075

\begin{abstract}
Cells of higher eukaryotes process double strand breaks (DSBs) in their genome using a non-homologous end joining apparatus that utilizes DNA-PK and other well characterized factors (D-NHEJ). Cells with defects in D-NHEJ, repair the majority of DSBs using a slow-repair pathway which is independent of genes of the RAD52 epistasis group and functions as a backup (B-NHEJ). Recent studies implicate DNA ligase III, PARP-1 and histone H1 in this pathway of NHEJ. The present study investigates the operation of B-NHEJ in the repair of interphase chromosome breaks visualized in irradiated $\mathrm{G}_{0}$ human lymphocytes by premature chromosome condensation (PCC). Chromosome breaks are effectively repaired in human lymphocytes, but repair is significantly compromised after treatment with wortmannin, a DNA-PK inhibitor. Despite slower kinetics, cells exposed to wortmannin rejoin the majority of IR induced chromosome breaks suggesting that B-NHEJ is also functional at the chromosome level. Complementation of D-NHEJ defect in wortmannintreated lymphocytes by newly made DNA-PK is only possible under conditions of nuclear envelope break down and premature chromosome condensation, suggesting that in interphase cells the shunting of chromosome breaks from DNHEJ to B-NHEJ is irreversible. The understanding of chromosomal aberration formation allows mechanistic explanations for the carcinogenic potential of D-NHEJ defects.
\end{abstract}

Correspondence to: Dr Gabriel E. Pantelias, Institute of Radioisotopes and Radiodiagnostic Products, National Centre for Scientific Research 'Demokritos', 15310 Ag. Paraskevi Attikis, Athens, Greece

E-mail: gabriel@ipta.demokritos.gr, or

Dr George Iliakis, Institute of Medical Radiation Biology, University of Duisburg-Essen Medical School, D-45122 Essen, Germany

E-mail: georg.iliakis@uk-essen.de

Key words: ionizing radiation, premature chromosome condensation, DNA-PK, chromosome damage, DNA repair, wortmannin

\section{Introduction}

Endogenous cellular processes and exogenous factors such as ionizing radiation (IR) can generate double strand breaks (DSB) in the DNA that undermine genomic integrity. Three fundamentally distinct processes, homologous recombination repair (HRR), single strand annealing (SSA), and nonhomologous end joining (NHEJ) can, in principle, repair DNA DSBs (1-5). In cells of higher eukaryotes, repair of ionizing radiation (IR) induced DSBs is dominated by a fast component operating with half times of a few minutes. This repair component is severely compromised by defects in any of the constituents of DNA-PK (6-8), as well as by defects in DNA ligase IV (9). We proposed the term D-NHEJ, for this DSB repair pathway to signify its dependence on DNA-PK and to discriminate it from other repair pathways $(7,9,10)$ (see below).

Despite the prevalence of D-NHEJ, reports by us and others indicate that cells with defects in any of its components are rejoining the majority of IR-induced DNA DSBs utilizing an alternative pathway operating with much slower kinetics $(6,8-10)$. Because HRR operates in yeast with much slower kinetics than D-NHEJ in higher eukaryotes, it was in principle possible that the slow component observed after inhibition of D-NHEJ reflects this process. We tested this hypothesis using the hyper-recombinogenic DT40 chicken cell line and a set of mutants defective in homologous recombination (HR) $(11,12)$. DT40 cells rejoin IR-induced DSBs with kinetics similar to those of other vertebrate cells displaying 1000-fold lower levels of HR (7). In addition, knockouts of RAD51B, $R A D 52$ and $R A D 54$ rejoin DSB with kinetics similar to the wild-type, as does also a conditional knock out mutant of $R A D 51$ (7). While a significant reduction in the fast component of rejoining is observed in $K u 70^{-/-}$DT40 cells, a double mutant $K u 70^{-/ / R A D} 54^{-/}$shows half times similar to $\mathrm{Ku} 70^{-/-}$cells (7).

Thus, increases by several orders of magnitude in the capacity of DT40 cells for HRR, or defects in the proteins involved, fail to alter their repair kinetics in a way compatible with a substantial involvement of HRR in the slow component of DNA DSBs rejoining, even when D-NHEJ is severely compromised (7). Furthermore, rejoining of DNA DSBs with slow kinetics is associated with the joining of incorrect ends (13), as well as with the extensive development of chromosome aberrations in the form of chromosome exchanges 
(14-16). These results are not compatible with a contribution from HRR, which is known to operate in a largely error-free manner. To accommodate these observations, we proposed that the slow component of DSB rejoining reflects an alternative pathway of NHEJ, which we termed backup, B-NHEJ - to differentiate from D-NHEJ and to indicate its backup role. According to this model, at least two distinct NHEJ pathways cooperate to remove IR-induced DNA DSBs from the genome of higher eukaryotes $(6,7,9,10,17,18)$. Recent work suggests that the PARP-1/DNA ligase III/XRCC1 module is involved in B-NHEJ (19-22), possibly supported by histone H1 (23). Furthermore, B-NHEJ is suppressed by D-NHEJ (24), shows cell cycle dependence with a more pronounced function during the $\mathrm{G}_{2}$ phase (25), and may be compromised in noncycling cells (26). A separate line of investigation provides solid genetic evidence for the involvement of backup end joining pathways in Ig class switch $(27,28)$ and V(D)J recombination (29).

The characterization of backup pathways of NHEJ has important implications for our understanding of the cellular responses to DNA damage and their importance in genomic stability $(10,30)$. It allows mechanistic explanations for the carcinogenic potential of D-NHEJ defects, and the development of models for chromosome aberration formation in irradiated cells (31). Although B-NHEJ is well documented at the DNA level, its contribution to the repair of interphase chromosome breaks is less well documented with only one early report showing defects in the repair of chromosome breaks in DNA-PKcs deficient cells (32). On the other hand, the extensive formation of exchange type aberrations, which of course requires end joining activities in cells defective in D-NHEJ (14-16), not only suggests the operation of such pathways at the chromosome level but it also demonstrates their error prone nature.

To directly address the operation of B-NHEJ at the chromosome level, we employed premature chromosome condensation (PCC) (33-36). In PCC, fusion of the interphase cell under examination (mainly cells in $\mathrm{G}_{1}$ or $\mathrm{G}_{2}$ phase of the cell cycle) with a non-irradiated metaphase cell causes the pre-mature condensation of the interphase chromosomes and allows direct examination of their integrity. In this way, and unlike analysis at metaphase, which only reports the final misrepair outcome, information on the actual repair kinetics of chromosome breaks can be obtained. In addition, experiments can be designed to examine the contribution of specific pathways to chromosome repair. This approach extends and complements global DSB repair studies by restricting the analysis to the DSB subset causing chromosome breaks. It is likely that this subset of DSBs is responsible for some of the most severe effects of radiation including the formation of lethal chromosome aberrations, or of cancer promoting genome rearrangements (31).

The experiments focus on effects measured in peripheral blood lymphocytes (PBL). PBLs are an ideal model for such studies due to their uniform accumulation in $\mathrm{G}_{0}$ phase, which facilitates cytogenetic analysis by PCC (35). The results obtained demonstrate the operation of backup pathways in the repair of chromosome breaks and point to interesting connections between mitotic events and complementation of DNA-PK defects.

\section{Materials and methods}

Cell culture and irradiation conditions. Experiments were carried out using peripheral blood lymphocytes (PBL) of normal volunteer donors. The lymphocytes were isolated from freshly drawn blood using Ficoll-Paque sedimentation and suspended in McCoy's 5A growth medium supplemented with $10 \%$ fetal bovine serum. Isolated lymphocytes were irradiated with a dose of $6 \mathrm{~Gy}$ at room temperature and at a dose rate of $1 \mathrm{~Gy} / \mathrm{min}$, using a GammaCell 220 irradiator (Atomic Energy of Canada Ltd., Ottawa, Canada).

Chinese hamster ovary $(\mathrm{CHO})$ cells were grown in McCoy's 5A culture medium supplemented with $10 \%$ fetal calf serum and antibiotics. Mitotic $\mathrm{CHO}$ cells were obtained from exponentially growing cells after a 4-h treatment with $0.2 \mu \mathrm{g} / \mathrm{ml}$ colcemid and harvested by selective detachment.

Cell fusion and premature chromosome condensation. The chromosomal damage under the various experimental conditions was measured in interphase lymphocytes using polyethylene glycol (PEG) mediated cell fusion and premature chromosome condensation (PCC) induction $(34,37)$. Briefly, $\mathrm{G}_{0}$ lymphocytes isolated from peripheral blood were fused to mitotic PCC-inducer CHO cells either immediately after irradiation or after incubation for repair for different times, in the presence or absence of wortmannin. For this purpose, mitotic cells and lymphocytes were washed separately with serum-free McCoy's 5A medium and mixed in a ratio of $\sim 1: 5$ in a $14-\mathrm{ml}$ round-bottom culture tube. After centrifugation at $200 \mathrm{~g}$ for $5 \mathrm{~min}$, the supernatant was discarded and $0.15 \mathrm{ml}$ of $50 \%(\mathrm{w} / \mathrm{v})$ PEG (PEG 1500, Roche), prepared in phosphate buffered saline (PBS), was quickly added and held for $\sim 1 \mathrm{~min}$. Subsequently, $1.5 \mathrm{ml}$ PBS was slowly added, the tube was gently shaken and the cell suspension centrifuged. The supernatant was discarded and the pellet resuspended in $0.7 \mathrm{ml}$ of McCoy's $5 \mathrm{~A}$ growth medium supplemented with colcemid $\left(0.05 \mathrm{ml}\right.$ from a $10^{-5} \mathrm{M}$ stock solution). After $75 \mathrm{~min}$ at $37^{\circ} \mathrm{C}$, cell fusion and PCC induction was completed and chromosome spreads were prepared by standard cytogenetic procedures. Routinely, 30-50 lymphocytes in $\mathrm{G}_{0}$ phase were scored for excess, $>46$, chromosome fragments for each experimental point. Mean values and standard deviations were obtained from three independent experiments.

Whole cell extract (WCE) preparation and DNA-PK assay. Lymphocytes were cultured for three days as described above. Cells were collected by centrifugation and washed in ice-cold PBS and subsequently washed with five packed cell volumes of cold hypotonic buffer (10 mM HEPES, $5 \mathrm{mM}$ $\mathrm{KCl}, 1.5 \mathrm{mM} \mathrm{MgCl}{ }_{2}, 0.2 \mathrm{mM}$ Phenylmethylsulphonyl fluoride and $0.5 \mathrm{mM}$ DTT). The pellet was resuspended in two volumes of hypotonic buffer and cells were disrupted by three cycles of freezing and thawing in liquid nitrogen and a $37^{\circ} \mathrm{C}$ water bath, respectively. Subsequently, $3 \mathrm{M} \mathrm{KCl}$ was slowly added to the homogenate to a final concentration of $0.5 \mathrm{M} \mathrm{KCl}$ and kept in ice for $30 \mathrm{~min}$, while frequently agitating. Subsequently, samples were centrifuged for $40 \mathrm{~min}$ at $14,000 \mathrm{rpm}$ at $4^{\circ} \mathrm{C}$ and the supernatant was aliquoted, snap-frozen and stored at $-80^{\circ} \mathrm{C}$. 
DNA-PK activity was measured as previously described (38). The peptide EPPLSQEAFADLWKK, corresponding to 11-24 amino acids of human p53 with threonine 18 and serine 20 changed to alanine was used as substrate. Five $\mu \mathrm{g}$ of WCE was mixed with $200 \mu \mathrm{M}$ of peptide, with or without $10 \mu \mathrm{g} / \mathrm{ml}$ sonicated calf thymus DNA in a volume of $18 \mu 1$ buffer containing $50 \mathrm{mM}$ HEPES (pH 7.5), $10 \mathrm{mM} \mathrm{MgCl}_{2}$, $50 \mathrm{mM} \mathrm{KCl}$ and $0.2 \mathrm{mM}$ EGTA. A radioactively labeled ATP solution was made by mixing $1 \mu 1$ of $\gamma$-[ $\left.{ }^{32} \mathrm{P}\right]$-ATP $(3,000 \mathrm{Ci} / \mathrm{mmol}, 10 \mu \mathrm{C} / \mu \mathrm{l})$ in $49 \mu 1$ unlabeled $5 \mathrm{mM}$ ATP. The reaction was started by adding $2 \mu \mathrm{l}$ of radioactively labeled ATP solution and was incubated at $30^{\circ} \mathrm{C}$ for $15 \mathrm{~min}$. The reaction was stopped by adding $20 \mu 1$ stop solution (30\% acetic acid, $1 \mathrm{mM}$ ATP). Subsequently, $20 \mu \mathrm{l}$ of the reaction mixture was spotted on Whatman p81 phosphocellulose paper and washed four times in $10 \%$ acetic acid for $15 \mathrm{~min}$. ${ }^{32} \mathrm{P}$ incorporation was measured by Kodak phosphoscreen, scanned with 'Typhoon' (GE Healthcare) and signal was analyzed by ImageQuant 5.2 Software (GE Healthcare).

Pulsed-field gel electrophoresis. Repair of DNA DSBs was evaluated by asymmetric field inversion gel electrophoresis (AFIGE), a pulsed-field gel electrophoresis method, according to previously published protocols (6). Cells were irradiated with a Pantak X-ray machine operated at $320 \mathrm{kV}, 10 \mathrm{~mA}$ with a $1.65 \mathrm{~mm} \mathrm{Al}$ filter (effective photon energy, $90 \mathrm{kV}$ ), at a dose rate of $2.7 \mathrm{~Gy} / \mathrm{min}$, at $50-\mathrm{cm}$ distance. To inhibit DNAPK activity, cells were incubated with $20 \mu \mathrm{M}$ Wortmannin (Sigma) 15-30 min prior to irradiation. Cells were cooled to $4^{\circ} \mathrm{C}$ prior to irradiation and were irradiated on ice. After irradiation, the medium was replaced with fresh growth medium preheated to $42^{\circ} \mathrm{C}$ (to rapidly restore $37^{\circ} \mathrm{C}$ ) and cells were returned to the incubator at $37^{\circ} \mathrm{C}$ to allow for repair. When appropriate, wortmannin at $20 \mu \mathrm{M}$ was also present in these media and was added to the cultures according to the indicated protocol. Cells were harvested at various times thereafter and embedded in agarose blocks containing $\sim 1 \times 10^{5}$ cells/block. AFIGE was carried out in $0.5 \%$ molecular biology grade agarose (Bio-Rad). The gels were prepared with $0.5 \mu \mathrm{g} / \mathrm{ml}$ ethidium bromide, and were run in $0.5 \mathrm{X} \mathrm{TBE}$ at $10^{\circ} \mathrm{C}$ for $40 \mathrm{~h}$. During this time, cycles of $1.25 \mathrm{~V} / \mathrm{cm}$ for $900 \mathrm{sec}$ in the direction of DNA migration alternated with cycles of $5.0 \mathrm{~V} / \mathrm{cm}$ for $75 \mathrm{sec}$ in the reverse direction. Signal was detected using the 'Typhoon' (GE Healthcare) and the fraction of DNA released (FDR) out of the well into the lane was quantified with the ImageQuant 5.2 Software (GE Healthcare). Repair kinetics was commonly plotted as FDR versus time. However, because the dependence of FDR on dose was not purely linear in the range of doses examined, a quantitative analysis of the repair kinetics could not be carried out with accuracy from such plots. To circumvent this problem, repair kinetics are shown here as plots of equivalent dose (Deq), measured in Gy, versus time (19). For this purpose, we calculated for FDR values obtained at each repair time-point a corresponding dose (Deq) from a dose response curve (FDR versus dose) generated in the same experiment with cells processed for electrophoresis immediately after irradiation. This way of plotting provides more accurate repair kinetic results and minimizes possible variations between experiments (7).

\section{Results}

To investigate the putative function of B-NHEJ in the repair of IR-induced chromosome breaks, we adopted peripheral blood lymphocytes (PBLs) as a model system. PBLs from healthy individuals have a normal chromosome complement and are devoid of cytogenetic and genetic alterations encountered in tumor cells, which may modify radiation response. In addition, unstimulated PBLs are uniformly and stably arrested in the $G_{0}$ phase of the cell cycle, which is very helpful for experiments analyzing interphase chromosome damage by means of PCC (34).

To analyze repair of IR induced interphase chromosome breaks, we incubated at $37^{\circ} \mathrm{C}$ for different times PBLs exposed to 6 Gy X-rays. Subsequently, we fused PBLs with mitotic $\mathrm{CHO}$ cells to induce premature condensation in the lymphocyte chromosomes and processed the samples for scoring. Fig. 1A shows representative spreads of human prematurely condensed chromosomes from cultures exposed to 0 or $6 \mathrm{~Gy}$ and analyzed immediately, 1 or $6 \mathrm{~h}$ later. IR-induced chromosome breakage is evident as an increase, $>46$, in the number of chromosome entities, which we refer here as excess chromosome fragments (ECFs). The number of ECFs is maximal in cells analyzed immediately after IR (20 in Fig. 1A) and drops progressively as cells are allowed to repair at $37^{\circ} \mathrm{C}$. Fig. $1 \mathrm{~B}$ summarizes the repair kinetics as ECFs versus repair time. Under the experimental conditions employed, 18 ECFs are scored per cell immediately after IR, reflecting an initial yield of 3 chromosome breaks per cell per Gy. PBLs effectively repair chromosome breaks and the number of ECFs drops within $6 \mathrm{~h}$ to $\sim 5$ per cell.

It is widely accepted that interphase chromosome breaks result from unrepaired DNA DSBs. In higher eukaryotes, IR-induced DSBs are mainly repaired by D-NHEJ (see Introduction), and it is reasonable to hypothesize that the same pathway contributes to the repair of chromosome breaks as well. IR induces 20-40 DSBs per Gy but, as shown here, only 2-4 interphase chromosome breaks, suggesting that only a subset of DSBs leads to chromosome breaks. The subset of DSBs leading to chromosome breaks is unlikely to be selected randomly from the entire pool of induced DSBs, as chromosome breaks are typically repaired with half times of about 1-2 h (Fig. 1B), whereas the majority of DSBs is repaired with half times of 5-20 min. Thus, only subsets of DSBs characterized by slow repair kinetics are likely to cause the chromosome breaks uncovered by PCC. These subsets of DSBs may be repaired by D-NHEJ, but it is equally possible that other pathways are involved in their repair as well. Indeed, as outlined in the Introduction, it is well documented that cells with defects in components of D-NHEJ remove the majority of DSBs with slow kinetics using B-NHEJ.

To evaluate the contribution of D-NHEJ to the repair of interphase chromosome breaks in PBLs and to examine whether B-NHEJ is capable of processing DSBs leading to chromosome breaks, we studied the effect of wortmannin on the repair of interphase chromosome breaks. Wortmannin is a non-competitive, irreversible inhibitor of PI 3-kinase that inhibits at higher concentrations other members of this kinase family including DNA-PKcs $(39,40)$. This causes a pronounced inhibition in the repair of IR induced DSBs by D-NHEJ (9). 
A

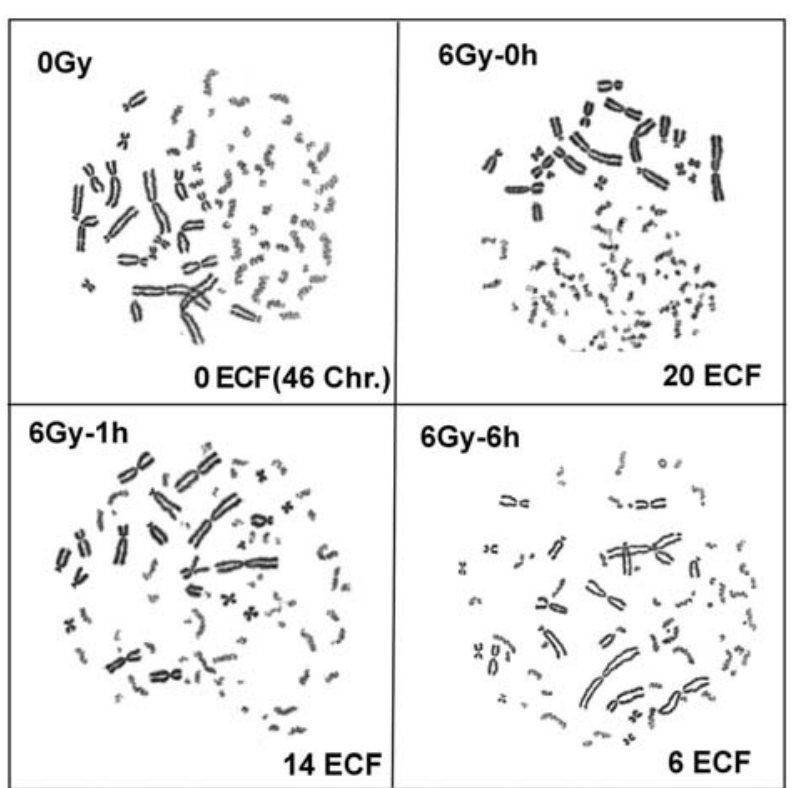

B

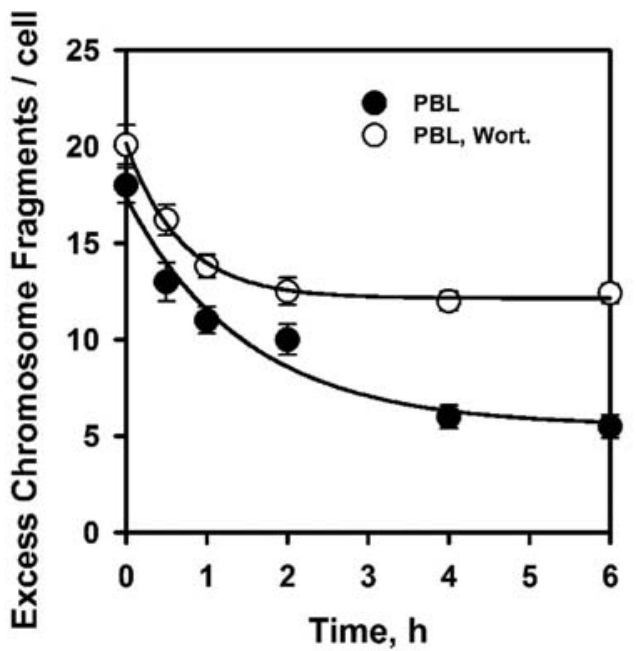

Figure 1. Wortmannin inhibits repair of chromosome breaks in PBLs. A. Micrographs of PBLs fused by means of PEG with $\mathrm{CHO}$ mitotics and incubated at $37^{\circ} \mathrm{C}$ for $1 \mathrm{~h}$ to allow for PCC. Mitosis promoting factor (MPF) from the mitotic cell causes the premature condensation of the $G_{0}$ PBL chromosomes into distinct units amenable to integrity analysis. The PBL in the upper left micrograph is unirradiated and shows 46 chromosomes as expected. Following exposure to $6 \mathrm{~Gy}$ and immediate analysis, 66 chromosome entities are scored corresponding to an excess chromosome fragment (ECF) number of 20 (upper right). After a 1-h postirradiation incubation time for repair, 14 ECF are scored in a PBL exposed to 6 Gy (lower left). This number is further reduced after $6 \mathrm{~h}$ of repair (lower right). B, Kinetics of repair of interphase chromosome breaks in PBLs that were either irradiated and incubated for repair at $37^{\circ} \mathrm{C}$ for the indicated times (filled circles), or irradiated and incubated for repair in the presence of $20 \mu \mathrm{M}$ wortmannin (open circles). Plotted is the number of ECF as a function of the repair time. The mean and standard error from three experiments are shown.

We reasoned that inhibition by wortmannin of DNA-PK in PBLs would allow, on the one hand, the evaluation of the contribution of D-NHEJ to the repair of chromosome breaks and, on the other hand, the detection of alternative repairpathways that gain impact when D-NHEJ is compromised (see Introduction). For these experiments, PBLs are incubated with $20 \mu \mathrm{M}$ wortmannin for $30 \mathrm{~min}$, exposed to IR and returned to $37^{\circ} \mathrm{C}$ for the indicated times. Fig. 1B shows the chromosome repair kinetics measured under these conditions. Wortmannin markedly slows down repair, demonstrating thus a contribution of D-NHEJ to the repair chromosome breaks in irradiated but untreated cells. However, despite marked inhibition, chromosome breaks are repaired to a significant extent presumably by alternative repair pathways. These results are qualitatively similar to those reported earlier using the DNA-PKcs deficient mouse scid cells (32).

Of note in the above experiment is the modest increase in the initial yield of IR-induced chromosome breaks in wortmannin-treated PBLs. Since there is no incubation for repair in this sample, the observed increase suggests inhibition by wortmannin of chromosome break repair occurring during the period of premature condensation of PBL chromosomes. Although of small magnitude in this type of experiments, the effect points to undetected activity of chromosome break repair [see also (41-43)] and raises questions regarding the validity of the interpretation given above.

To determine the contribution of D-NHEJ to chromosome repair during incubation for chromosome condensation, PBLs were treated with wortmannin and analyzed in PCC assays also carried out in the presence of wortmannin $(20 \mu \mathrm{M})$. Fig. 2A shows typical examples of chromosome spreads obtained under these conditions. Wortmannin has no detectable clastogenic effect on unirradiated cells, and both irradiated and non-irradiated PBLs show after treatment with wortmannin levels of chromatin condensation similar to those of untreated cells. Fig. 2B summarizes chromosome break repair kinetics in wortmannin-treated PBLs and analyzed in PCC reactions containing wortmannin as well. A significant increase in the initial yield of chromosome breaks is observed substantiating D-NHEJ activity during chromosome conden-sation. At the same time, marked repair is still evident, further implicating B-NHEJ in the rejoining of chromosome breaks when DNAPK activity is compromised.

The sensitivity to wortmannin of the initial yield of chromosome breaks suggests wortmannin-sensitive repair during PCC and therefore residual DNA-PK activity. It seemed unlikely that the source of this DNA-PK activity is the PBL, as treatment with $20 \mu \mathrm{M}$ wortmannin should largely inactivate the existing DNA-PK pool (see below). On the other hand, DNA-PK is present in the mitotic CHO cell and may complement wortmannin treated-PBLs during incubation for premature chromosome condensation. Indeed, this cell fusion approach has been previously used to establish complementation groups among D-NHEJ mutants (44). To examine this possibility, mitotic cells were collected from $\mathrm{CHO}$ cultures that were pre-treated for $1 \mathrm{~h}$ with $20 \mu \mathrm{M}$ wortmannin and fused with PBLs to induce PCC in reactions also carried out in the presence of wortmannin. The results obtained are summarized in Fig. 2B. A marked increase, over that of reactions assembled with wortmannin, is observed in the number of excess chromosome fragments, demonstrating that mitotic DNA-PK activity is a key mediator of D-NHEJdependent chromosome repair during PCC. This observation makes also evident that simple administration of wortmannin during PCC reaction-assembly is not inhibiting in a timely fashion the function of mitotic DNA-PK. Notably, even under conditions of such wide inhibition of DNA-PK activity, 
A

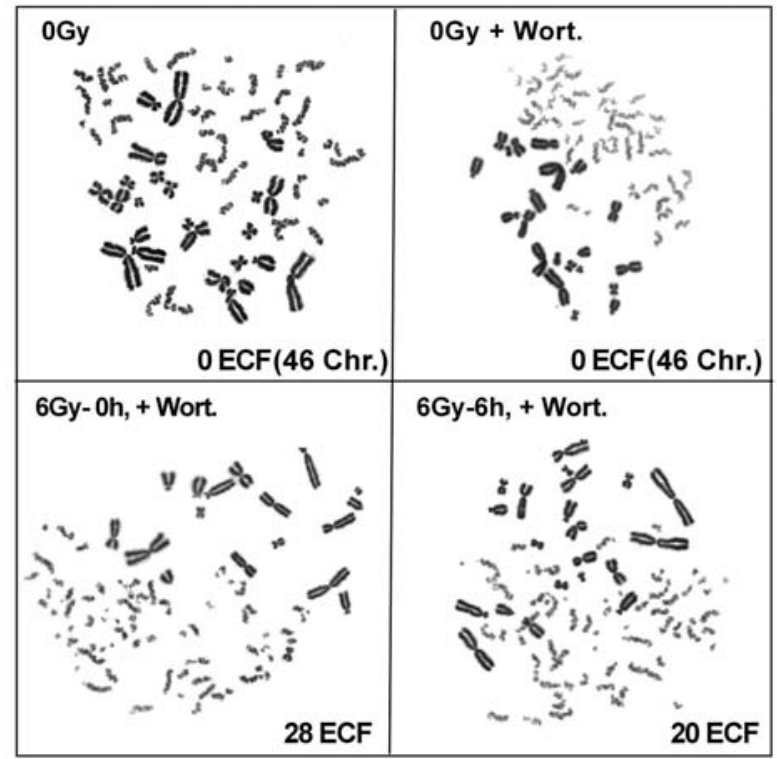

B

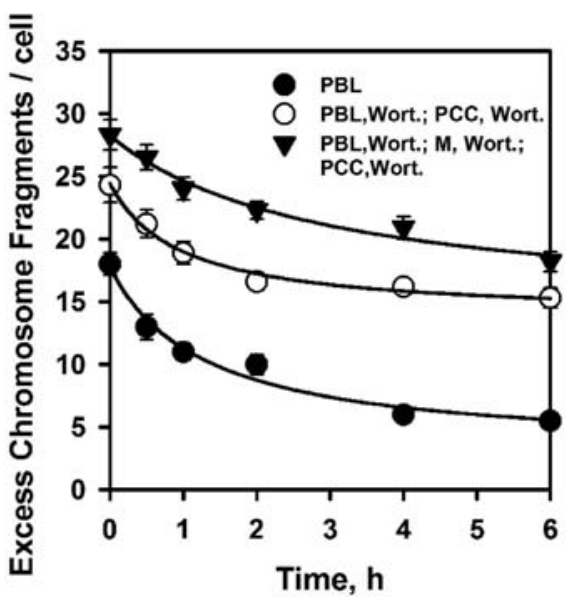

Figure 2. DNA-PKcs from CHO mitotics contributes to chromosome repair in wortmannin treated PBLs. A, Micrographs of PBLs fused to $\mathrm{CHO}$ mitotics after different treatments. The upper left micrograph shows a syssitium between an unirradiated PBL and a $\mathrm{CHO}$ mitotic, whereas the upper right micrograph a syssitium between an unirradiated, wortmannin-treated PBL and a CHO mitotic. The lower left micrograph shows a syssitium with a PBL treated with wortmannin and exposed to $6 \mathrm{~Gy}$, whereas the lower right panel a syssitium with a PBL irradiated and treated with wortmannin for $6 \mathrm{~h}$. Note the increase in ECF as compared to a similarly treated PBL in Fig. 1. $\mathrm{B}$, Kinetics of chromosome repair in untreated PBLs (filled circles), in PBLs treated with wortmannin before IR and during incubation for PCC (open circles), as well as with PBLs treated with wortmannin before irradiation and then fused for PCC in the presence of wortmannin with mitotic cells obtained from $\mathrm{CHO}$ cultures that were also treated with wortmannin (filled triangles). Plotted is the number of ECF as a function of the repair time. The mean and standard error from three experiments are shown.

chromosome break repair activity is amply documented, further underlining the putative function of B-NHEJ.

To better evaluate the contribution of B-NHEJ to chromosome repair when D-NHEJ is compromised, we followed repair of interphase chromosome breaks for up to $48 \mathrm{~h}$ in untreated PBLs, as well as in PBLs treated with wortmannin using different protocols. Fig. 3A summarizes the results obtained. As expected, effective repair is observed in untreated PBLs. On the other hand, the initial yield of chromosome
A

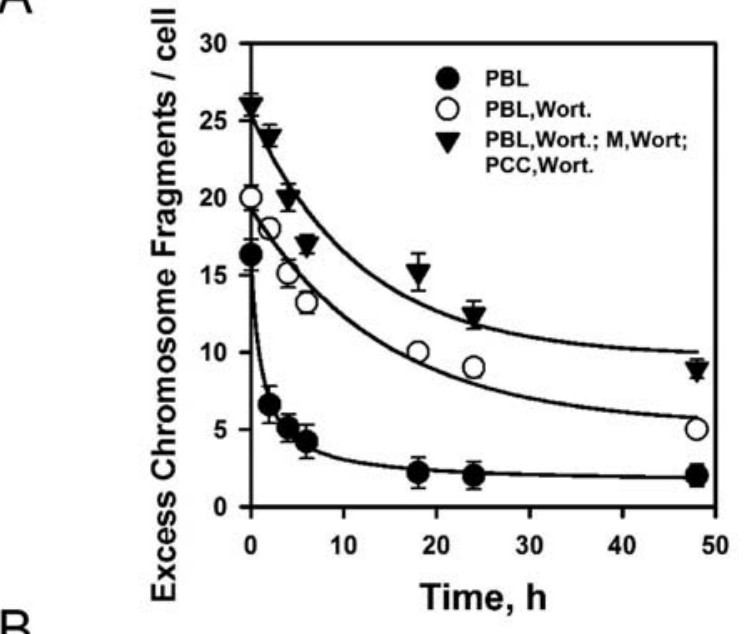

B

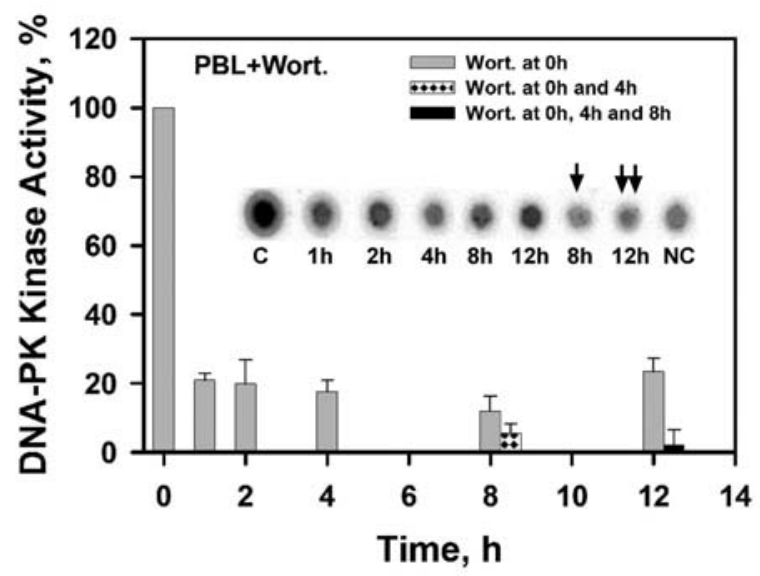

Figure 3. Efficient repair of chromosome breaks under conditions inhibiting DNA-PK. A, Kinetics of chromosome repair as measured by PCC in irradiated PBLs. Filled circles show the results obtained with PBLs incubated for repair at $37^{\circ} \mathrm{C}$ in the absence of any treatment. Open circles show results obtained with PBLs that were treated once with wortmannin before IR. Filled triangles show results obtained with PBLs treated with wortmannin before IR as well as at 4-h intervals thereafter. B, DNA-PK activity measured as described in Materials and methods in PBLs at various times after treatment with $20 \mu \mathrm{M}$ wortmannin. Included in the figure are also results of PBLs treated with wortmannin repeatedly at 4-h intervals.

breaks is markedly increased and their repair compromised after a single treatment with wortmannin when PCC is carried out in the presence of wortmannin using wortmannintreated mitotic cells (filled triangles). Despite this increase in yield and decrease in rate, a large proportion of chromosome breaks is repaired within $48 \mathrm{~h}$, in line with the operation of B-NHEJ.

In the above experiment, which lasted a total of $48 \mathrm{~h}$, PBLs were treated with wortmannin only once before irradiation. It is therefore conceivable that the results obtained, particularly after long repair times, include D-NHEJ supported by residual DNA-PK activity, as well as by newly synthesized DNA-PK. To examine this possibility, we carried out experiments in which wortmannin was repeatedly added (every 3-5 h) to PBLs and repair of chromosome breaks was followed. Repeated drug addition ensures that residual DNA-PK activity will be further inhibited and that newly made activity will be compromised. Repair of chromosome breaks 
A

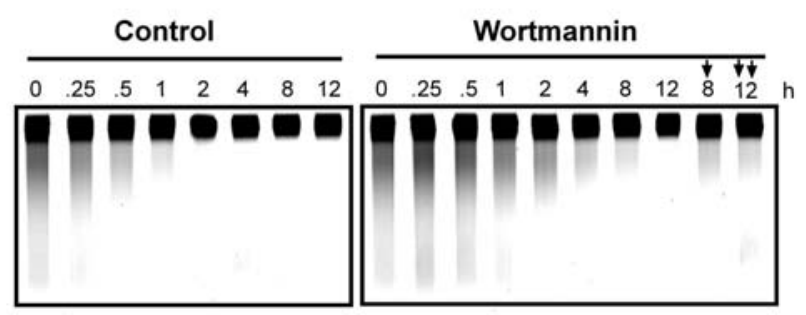

B

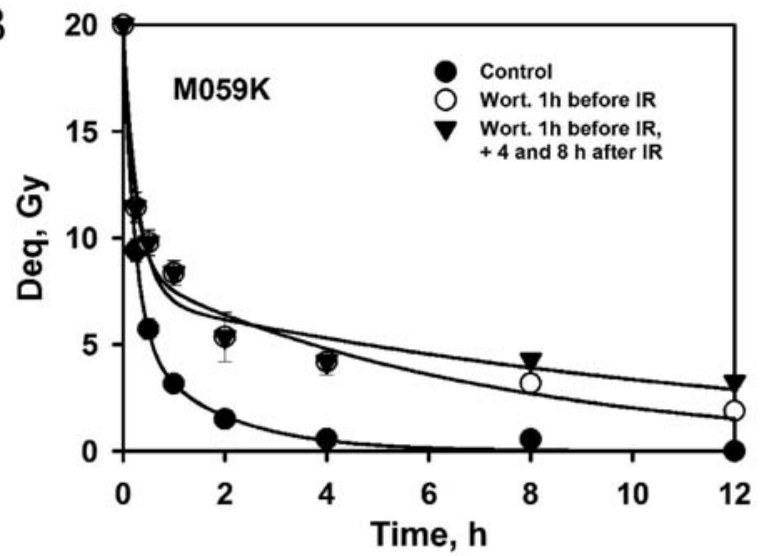

Figure 4. Repair of DNA DSBs in M059K cells exposed to wortmannin under different conditions. Exponentially growing M059K cells were exposed to $20 \mathrm{~Gy}$ and repair of DSBs measured using pulsed-field gel electrophoresis, as outlined in Materials and methods. A, Typical gels of control and wortmannin-treated cells. An asterisk indicates samples repeatedly treated with wortmannin. B, Quantitative analysis of PFGE results. For repair cells were either left untreated (filled circles), or were treated with $20 \mu \mathrm{M}$ wortmannin once before irradiation (open circles). Included are also results obtained with cells treated with wortmannin every $4 \mathrm{~h}$ (filled triangles). Plotted is Deq, a parameter describing residual load of DSBs, as a function of time. The results shown represent the mean and standard error from three determinations.

measured under these conditions is included in Fig. 3. It is evident that repair kinetics similar to those obtained after a single drug addition are observed suggesting that inhibition of residual, or newly made DNA-PK has no detectable effect on the kinetics of chromosome repair, even after long incubation times. The lower initial and subsequent yield of chromosome breaks in this experiment, as compared to that shown by the filled triangles, is due to the fact that wortmannin was not applied to the mitotics or during fusion. This is intriguing because it suggests that DNA-PK activity provided during PCC can assist chromosome repair, whereas DNA-PK activity generated in the interphase cell lacks this potential.

To validate the rationale of the above experiment, we measured DNA-PK activity in similarly treated PBLs and the results obtained are summarized in Fig. 3B. After a single treatment with wortmannin DNA-PK activity drops precipitously but the measurements indicate some residual activity. This residual activity, and possibly also the activity of newly made DNA-PK, are compromised by repeated addition of wortmannin as expected. The lack of contribution to chromosome repair of residual and newly synthesized DNA-PK suggests that chromosome breaks are irrevocably shunted to alternative pathways of repair when D-NHEJ is compromised by the first application of wortmannin. On the other hand, the observation that DNA-PK of mitotic cells contributes equally to chromosome repair at all time-points examined (compare open circles and filled triangle) suggests that mitotic events, such as those induced during PCC (nuclear membrane break down and chromatin condensation), generate opportunities for DNA-PK function in a subset of chromosome breaks.

While the results presented in Fig. 3 suggest that residual or even newly synthesized DNA-PK is unable to contribute to chromosome repair once breaks are shunted in alternative repair pathways, it is unclear whether this holds for all DSBs or just for the subset underlying chromosome breaks. To address this question, we carried out experiments in which M059K cells, a human tumor cell line, were treated with wortmannin either once before irradiation, or repeatedly (every $4 \mathrm{~h}$ ), and DSB repair was measured by PFGE. Fig. 4 summarizes the results obtained. While a single treatment with wortmannin markedly inhibits DSB rejoining, repeated addition of the inhibitor does not produce any additional effect. This result suggests that not only DSBs underlying chromosome breaks, but also the bulk of DSBs are shunted irrevocably to alternative repair pathways leaving newly synthesized DNA-PK unable to regain control.

\section{Discussion}

D-NHEJ is a major contributor of chromosome repair. The results presented above demonstrate extensive contribution of D-NHEJ to the repair of interphase chromosome in PBLs. Because PBLs are directly derived from healthy individuals, the results attest to the function of this pathway in chromosome break repair in a model system maintaining genomic stability. Although this observation should be regarded as largely expected on the basis of the extensive contribution of D-NHEJ to DSB repair, it is important to emphasize that interphase chromosome breaks reflect only a small $(\sim 10 \%)$ subset of DNA DSBs and may therefore arise as a result of the specific properties of this DSB subset - biochemical nature or localization in chromatin. These properties might indeed cause a shunting of these DSBs to other repair pathways such as HRR. Other reports point also to the induction by IR of a subset $(\sim 15 \%)$ of DSBs displaying ATM/Artemis requirement for their repair, presumably due to their complexity (45). Therefore, the observation that repair of IR-induced chromosome breaks extensively utilizes DNA-PK, points to a level of utilization of D-NHEJ for this subset of DSBs that is roughly similar to the level of utilization of D-NHEJ for the bulk of DSBs. A significant contribution of D-NHEJ to chromosome repair has also been reported in studies evaluating interphase chromosome repair in DNA-PK deficient mouse scid cells (32).

While the nature and the properties of DSBs giving rise to chromosome breaks in the premature chromosome condensation assay remain uncharacterized biochemically, the kinetics of chromosome break repair suggests that chromosome breaks derive from slowly repairing DSBs. Because slow rejoining kinetics is also a characteristic of B-NHEJ (see Introduction), it was theoretically possible that repair of interphase chromosome breaks predominantly 
reflects the function of B-NHEJ even in repair proficient cells in the absence of D-NHEJ inhibiting manipulations. This hypothesis can now be refuted by the sensitivity to wortmannin of chromosome break repair, which directly points to the involvement of DNA-PK.

The observations at the chromosome level reported here, combined with results of bulk DSB repair allow the conclusion that DNA-PK contributes to the repair of an entire spectrum of DSBs differing from each other in several important aspects that determine their kinetics of repair. It is important to characterize these subsets of DSBs and to determine the characteristic properties that define the cellular responses elicited by them. Experiments such as those described here utilizing the method of premature chromosome condensation may allow valuable contributions along these lines. For example, it will be particularly valuable to re-examine the contribution of ATM to the repair of interphase chromosome breaks $(33,36)$ in light of the recently proposed roles of ATM in the repair of subsets of complex DSBs (45).

$B$-NHEJ contributes to chromosome break repair when DNHEJ is compromised. The use of wortmannin to compromise D-NHEJ clearly demonstrates that PBLs recruit alternative pathways to repair chromosome breaks when the main DNA-PK dependent repair pathway is compromised. Indeed, even after inhibition of DNA-PK activity not only in the interphase PBLs but also in the mitotic CHO cells, substantial rejoining activity is detected able to remove from the genome the majority of IR-induced interphase chromosome breaks. Because the contribution of this repair activity is only evident after compromising D-NHEJ, it appears to serve a backup function (B-NHEJ) rather than the function of an equivalent, alternative repair pathway $(6,7,9,10,17,18)$. It is notable that repair of chromosome breaks by B-NHEJ proceeds significantly slower than by D-NHEJ and remains incomplete even after $24 \mathrm{~h}$ of postirradiation incubation. Assuming that the first determinant of repair kinetics is the characteristics of the DSB (chemical nature or location in chromatin - see above) the second determinant will be the repair pathway employed, with B-NHEJ operating slower than D-NHEJ on all subsets of DSBs induced.

Biochemical and genetic evidence implicate DNA ligase III and PARP-1 in the repair of DSBs by B-NHEJ (19-22). Since experiments demonstrating the contribution of these proteins are not part of this study, their potential involvement in chromosome repair should be regarded as tentative. In particular, we cannot formally exclude the operation in chromosome repair of other pathways such as HRR or single strand annealing, although the operation of the former in $G_{0}$ cells is considered unlikely.

$D$-NHEJ and B-NHEJ during PCC. When wortmannin is applied to mitotic cells, as well as during fusion for PCC, an increase in the initial yield of chromosome breaks is observed. This indicates that the presence of the inhibitor helps uncover chromosome breaks that would have been otherwise repaired during the incubation period for PCC. While the sensitivity to wortmannin of these chromosome breaks suggests the primary function of D-NHEJ in their repair, the extensive and nearly complete, albeit slow, chromo- some break repair measured when wortmannin is present in all components of the PCC reaction indicates that the same subset of chromosome breaks can also be processed by B-NHEJ.

The increase in the initial yield of chromosome breaks reported here in PCC reactions assembled with wortmannin is reminiscent of earlier results generated applying $\beta$-araA and hypertonic salt solution during PCC (41-43). These treatments, when applied during PCC, allow the characterization of two distinct subsets of interphase chromosome breaks. While $\beta$-araA-sensitive breaks are repaired with kinetics similar to those measured for the bulk of interphase chromosome breaks, hypertonic-treatment-sensitive DSBs are repaired with much faster kinetics, similar to those measured for the bulk of DSBs (41-43). Notably, mutants deficient in D-NHEJ such as $\mathrm{xrs}-5$, show resistance to hypertonic treatment suggesting that $\mathrm{Ku}$ deficiency specifically compromises repair of this subset of chromosome breaks. Furthermore, since $B$-araA is a DNA synthesis inhibitor, the results suggest that a subset of DSBs requiring DNA synthesis for repair underlies the $B$-araA sensitive subset of DSBs. Further experiments are required to reconcile these early observations with the results reported here with wortmannin and to generate a more complete model on the interplay of different pathways for the repair of DSBs and chromosome breaks.

Is the shunting of DSBs and chromosome breaks from DNHEJ to $B$-NHEJ irreversible? The rapid inactivation by hydrolysis of wortmannin in aqueous solutions enables the generation of new pools of DNA-PK by de novo protein synthesis. This new pool of DNA-PK, together with any residual DNA-PK activity, can in principle initiate DSB repair and thus also chromosome repair by D-NHEJ, particularly after long incubation times. Therefore, our observation that such pools of DNA-PK do not contribute to DSB or chromosome repair was unexpected and suggests the irreversible shunting of interphase chromosome breaks and even of the bulk of IR induced DSBs to B-NHEJ. This lack of contribution by fresh DNA-PK cannot be regarded as a consequence of a 'freezing' of the inhibited DNA-PK complex at the DNA ends, as observed in vitro (46), because DNA ends become available to the repair factors of B-NHEJ.

Thus, neither residual DNA-PK activity nor newly synthesized DNA-PK can have a detectable contribution to the repair of DSBs, once these lesions have been diverted to B-NHEJ. This shows, to our knowledge for the first time, that pathway selection is a one-way process with an irreversible character, once a decision is made. The biochemical processes underlying this aspect of repair pathway organization and mutual coordination remain to be established. However, it is likely that such aspects of repair pathway interaction will be important for the overall coordination of DSB repair and for the interactions between DNA repair and DNA damage checkpoints.

A notable exception to the irreversible shunting of DSBs to B-NHEJ after treatment with wortmannin seems to be generated during PCC. During this time period and independently of the elapsed postirradiation time interval, DNA-PK regains access to some unrepaired DSBs and removes them by D-NHEJ. Repair activity measured in this 
way is likely to take place during the initial stages of PCC, when the nuclear membrane of the interphase cell breaks down and chromosome condensation starts. It is tempting to speculate that under these conditions, changes in nuclear organization facilitate chromatin reprogramming that allow access of DNA-PK to DSBs. Drawing parallels with DNA replication (47), it may be argued that in this way chromatin gets licensed for D-NHEJ.

Exchange aberrations in DNA-PK deficient cells are compatible with B-NHEJ defect. Classical cytogenetic studies corroborate our conclusions of backup pathways of chromosome repair and provide direct evidence for their error-prone nature. Thus, DNA-PK deficient human tumor cells show a strong increase in exchange-type aberrations indicative of a DNA-PK-independent rejoining pathway, which by operating in an error-prone manner frequently lead to the formation of exchange-type aberrations (16). Similar observations have also been made in DNA-PK deficient MEFs (14). Also treatment with wortmannin of wild-type cells causes effects similar to those observed in DNA-PK mutants (15), suggesting that the inhibitor accurately recapitulates, in wild-type cells, the equivalent mutant phenotype.

The error-prone nature of backup pathways of NHEJ raises questions regarding its actual utility and biological significance. The expected increase in the frequency of exchange-type aberrations is likely associated with the generation of lethal events that will eliminate the affected cell even at relatively low damage loads. When non-lethal chromosome aberrations are generated, they are likely to cause chromosomal instability and cancer (see Introduction). A repair pathway whose function is associated with such negative effects is likely to be a liability for the cell and the organism and may offer a target for measures countering the development of cancer.

\section{Acknowledgements}

This study was supported by grants from the Hellenic Institute for Occupational Health and Safety, the German Research Foundation (DFG), as well as by Contract No. FIGH-CT-200200218 awarded by the European Commission.

\section{References}

1. Symington LS: Role of RAD52 epistasis group genes in homologous recombination and double-strand break repair. Microbiol Mol Biol Rev 66: 630-670, 2002.

2. Jackson SP: Sensing and repairing DNA double-strand breaks. Carcinogenesis 23: 687-696, 2002.

3. Doherty AJ and Jackson SP: DNA repair: how KU makes ends meet. Curr Biol 11: R920-R924, 2001.

4. Thompson LH and Schild D: Homologous recombinational repair of DNA ensures mammalian chromosome stability. Mutat Res 477: 131-153, 2001.

5. Haber JE: Gatekeepers of recombination. Nature 398: 665-667, 1999.

6. Di Biase SJ, Zeng Z-C, Chen R, Hyslop T, Curran WJ Jr and Iliakis G: DNA-dependent protein kinase stimulates an independently active, non-homologous, end-joining apparatus. Cancer Res 60: 1245-1253, 2000.

7. Wang H, Zeng Z-C, Bui T-A, Sonoda E, Takata M, Takeda S and Iliakis $\mathrm{G}$ : Efficient rejoining of radiation-induced DNA double-strand breaks in vertebrate cells deficient in genes of the RAD52 epistasis group. Oncogene 20: 2212-2224, 2001.
8. Nevaldine B, Longo JA and Hahn PJ: The scid defect results in much slower repair of DNA double-strand breaks but not high levels of residual breaks. Radiat Res 147: 535-540, 1997.

9. Wang H, Zhao-Chong Z, Perrault AR, Cheng X, Qin W and Iliakis G: Genetic evidence for the involvement of DNA ligase IV in the DNA-PK-dependent pathway of non-homologous end joining in mammalian cells. Nucleic Acids Res 29: 1653-1660, 2001 .

10. Iliakis G, Wang H, Perrault AR, Boecker W, Rosidi B, Windhofer F, Wu W, Guan J, Terzoudi G and Pantelias G: Mechanisms of DNA double strand break repair and chromosome aberration formation. Cytogenet Genome Res 104: 14-20, 2004.

11. Takata M, Sasaki MS, Sonoda E, Morrison C, Hashimoto M, Utsumi H, Yamaguchi-Iwai Y, Shinohara A and Takeda S: Homologous recombination and non-homologous end-joining pathways of DNA double-strand break. EMBO J 17: 5497-5508, 1998

12. Sonoda E, Sasaki MS, Buerstedde J-M, Bezzubova O, Shinohara A, Ogawa H, Takata M, Yamaguchi-Iwai Y and Takeda S: Rad51-deficient vertebrate cells accumulate chromosomal breaks prior to cell death. EMBO J 17: 598-608, 1998.

13. Löbrich M, Rydberg B and Cooper PK: Repair of X-ray-induced DNA double-strand breaks in specific Not I restriction fragments in human fibroblasts: joining of correct and incorrect ends. Proc Natl Acad Sci USA 92: 12050-12054, 1995

14. Martin M, Genesca A, Latre L, Jaco I, Taccioli GE, Egozcue J, Blasco MA, Iliakis G and Tusell L: Postreplicative joining of DNA double-strand breaks causes genomic instability in DNAPKcs-deficient mouse embryonic fibroblasts. Cancer Res 65: 10223-10232, 2005.

15. Virsik-Köpp P, Hofman-Hüther H, Rave-Fränk $M$ and Schmidberger H: The effect of wortmannin on radiation-induced chromosome aberration formation in the radioresistant tumor cell line WiDr. Radiat Res 164: 148-156, 2005.

16. Virsik-Köpp $P$, Rave-Fränk M, Hofman-Hüther $H$ and Schmidberger H: Role of DNA-PK in the process of aberration formation as studied in irradiated human glioblastoma cell lines M059K and M059J. Int J Radiat Biol 79: 61-68, 2003.

17. Wang H, Perrault AR, Takeda Y, Qin W, Wang H and Iliakis G: Biochemical evidence for Ku-independent backup pathways of NHEJ. Nucleic Acids Res 31: 5377-5388, 2003.

18. Asaad NA, Zeng Z-C, Guan J, Thacker J and Iliakis G: Homologous recombination as a potential target for caffeine radiosensitization in mammalian cells: reduced caffeine radiosensitization in XRCC2 and XRCC3 mutants. Oncogene 19: 5788-5800, 2000.

19. Windhofer F, Wu W and Iliakis G: Low levels of DNA ligases III and IV sufficient for effective NHEJ. J Cell Physiol 213: 475-483, 2007.

20. Wang M, Wu W, Wu W, Rosidi B, Zhang L, Wang $\mathrm{H}$ and Iliakis G: PARP-1 and Ku compete for repair of DNA double strand breaks by distinct NHEJ pathways. Nucleic Acids Res 34: 6170-6182, 2006.

21. Wang H, Rosidi B, Perrault R, Wang M, Zhang L, Windhofer F and Iliakis G: DNA ligase III as a candidate component of backup pathways of nonhomologous end joining. Cancer Res 65: 4020-4030, 2005.

22. Audebert M, Salles B and Calsou P: Involvement of poly(ADPribose) polymerase-1 and XRCC1/DNA ligase III in an alternative route for DNA double-strand breaks rejoining. J Biol Chem 279: 55117-55126, 2004.

23. Rosidi B, Wang M, Wu W, Sharma A, Wang H and Iliakis G: Histone $\mathrm{H} 1$ functions as a stimulatory factor in backup pathways of NHEJ. Nucleic Acids Res (In press).

24. Perrault R, Wang H, Wang M, Rosidi B and Iliakis G: Backup pathways of NHEJ are suppressed by DNA-PK. J Cell Biochem 92: 781-794, 2004.

25. Wu W, Wang M, Wu W, Singh SK, Mussfeldt T and Iliakis G: Repair of radiation induced DNA double strand breaks by backup NHEJ is enhanced in G2. DNA Repair 7: 329-338, 2008.

26. Windhofer F, Wu W, Wang M, Singh SK, Saha J, Rosidi B and Iliakis G: Marked dependence on growth state of backup pathways of NHEJ. Int J Radiat Oncol Biol Phys 68: 1462-1470, 2007.

27. Yan CT, Boboila C, Souza E K, Franco S, Hickernell TR, Murphy M, Gumaste S, Geyer M, Zarrin AA, Manis JP, Rajewsky K and Alt FW: IgH class switching and translocations use a robust non-classical end-joining pathway. Nature 449: 478-482, 2007. 
28. Soulas-Sprauel P, Le Guyader G, Rivera-Munoz P, Abramowski V, Olivier-Martin C, Goujet-Zalc C, Charneau P and De Villartay J-P: Role for DNA repair factor XRCC4 in immunoglobulin class switch recombination. J Exp Med 204: 1717-1727, 2007.

29. Corneo B, Wendland RL, Deriano L, Cui X, Klein IA, Wong S-Y, Arnal S, Holub AJ, Weller GR. Pancake BA, Shah S, Brandt VL, Meek K and Roth DB: Rag mutations reveal robust alternative end joining. Nature 449: 483-486, 2007.

30. Nussenzweig A and Nussenzweig MC: A backup DNA repair pathway moves to the forefront. Cell 131: 223-225, 2007.

31. Iliakis $\mathrm{G}, \mathrm{Wu} \mathrm{W}$, Wang M, Terzoudi GI and Pantelias GE: Backup pathways of non-homologous end joining may have a dominant role in the formation of chromosome aberrations. In: Chromosomal Alterations. Obe G and Vijayalaxmi (eds). Springer Verlag, Berlin, pp67-85, 2007.

32. Evans JW, Liu XF, Kirchgessner CU and Brown JM: Induction and repair of chromosome aberrations in scid cells measured by premature chromosome condensation. Radiat Res 145: 39-46, 1996.

33. Cornforth MN and Bedford JS: X-ray-induced breakage and rejoining of human interphase chromosomes. Science 222: 1141-1143, 1983.

34. Pantelias GE and Maillie HD: A simple method for premature chromosome condensation induction in primary human and rodent cells using polyethylene glycol. Somat Cell Genet 9: 533-547, 1983.

35. Pantelias GE and Maillie HD: The use of peripheral blood mononuclear cell prematurely condensed chromosomes for biological dosimetry. Radiat Res 99: 140-150, 1984.

36. Cornforth MN and Bedford JS: On the nature of a defect in cells from individuals with ataxia-telangiectasia. Science 227: 1589-1590, 1985.

37. Terzoudi GI, Manola KN, Pantelias GE and Iliakis G: Checkpoint abrogation in $\mathrm{G} 2$ compromises repair of chromosomal breaks in ataxia telangiectasia cells. Cancer Res 65: 11292-11296, 2005.

38. Lees-Miller SP, Sakaguchi K, Ullrich SJ, Appella E and Anderson CW: Human DNA-activated protein kinase phosphorylates serines 15 and 37 in the amino-terminal transactivation domain of human p53. Mol Cell Biol 12: 5041-5049, 1992.
39. Hartley KO, Gell D, Smith GC. M, Zhang H, Divecha N, Connelly MA, Admon A, Lees-Miller SP, Anderson CW and Jackson SP: DNA-dependent protein kinase catalytic subunit: a relative of phosphatidylinositol 3-kinase and the ataxia telangiectasia gene product. Cell 82: 849-856, 1995.

40. Blunt T, Finnie NJ, Taccioli GE, Smith GCM, Demengeot J, Gottlieb TM, Mizuta R, Varghese AJ, Alt F W, Jeggo PA and Jackson SP: Defective DNA-dependent protein kinase activity is linked to $\mathrm{V}(\mathrm{D}) \mathrm{J}$ recombination and DNA repair defects associated with the murine scid mutation. Cell 80: 813-823, 1995.

41. Iliakis G, Okayasu R, Varlotto J, Shernoff C: and Wang Y: Hypertonic treatment during premature chromosome condensation allows visualization of interphase chromosome breaks repaired with fast kinetics in irradiated CHO cells. Radiat Res 135: 160-170, 1993.

42. Okayasu R and Iliakis $\mathrm{G}$ : Ionizing radiation induces two forms of interphase chromosome breaks in $\mathrm{CHO}$ cells that rejoin with different kinetics and show different sensitivity to treatment in hypertonic medium or B-araA. Radiat Res 136: 262-270, 1993.

43. Okayasu R and Iliakis G: Evidence that the product of the $x r s$ gene is predominantly involved in the repair of a subset of radiation-induced interphase chromosome breaks rejoining with fast kinetics. Radiat Res 138: 34-43, 1993.

44. Darroudi F and Natarajan AT: Cytogenetical characterization of Chinese hamster ovary X-ray-sensitive mutant cells xrs5 and xrs6. Mutat Res 213: 249-255, 1989.

45. Riballo E, Kühne M, Rief N, Doherty A, Smith GC M, Recio M-J, Reis C, Dahm K, Fricke A, Krempler A, Parker AR, Jackson SP, Gennery A, Jeggo PA and Loebrich M: A pathway of doublestrand break rejoining dependent upon ATM, Artemis, and proteins locating to $\gamma-\mathrm{H} 2 \mathrm{AX}$ foci. Mol Cell 16: 715-724, 2004.

46. Calsou P, Frit P, Humbert O, Muller C, Chen DJ and Salles B: The DNA-dependent protein kinase catalytic activity regulates DNA end processing by means of Ku entry into DNA. J Biol Chem 274: 7848-7856, 1999.

47. Tada S and Blow JJ: The replication licensing system. Biol Chem 379: 941-949, 1998. 